\title{
Remoción de demanda química de Oxígeno de efluentes de remojo de curtiduría utilizando microalgas Chlorella sp. viva en suspensión
}

Removal of chemical Oxygen demand of tannery soak liquor using microalga Chlorella $s p$. live suspended

Jorge L. Mendoza Bobadilla', Melissa León Hurtado', José V. Nunja García², Ronald F. Rodríguez Espinoza ${ }^{2}$, Manuel Vera Herrera', Juan M. Ipanaqué Roña ${ }^{2}$

\section{RESUMEN}

Objetivo: Evaluar a escala de laboratorio la remoción de la demanda química de oxígeno utilizando microalga Chlorella saccharophila viva en suspensión, para el tratamiento de las aguas residuales provenientes de la etapa de remojo de curtiembre. Material y Métodos: Se utilizaron 3 biorreactores los cuales permitían la entrada de aire y luz solar, en los cuales se estableció diferentes relaciones de volúmenes entre la solución de microalga y el agua de remojo equivalentes a 3:1, 1:1 y 1:3, tomándose muestras cada 2 días para su posterior análisis de DQO en el Laboratorio de Investigación en Agua de la Universidad Nacional de Trujillo. Resultados: Los resultados muestran que el reactor de relación en volumen de 1:3 es el que logró remover el mayor porcentaje de DQO llegando a $90 \%$ en el día 14 de tratamiento, seguido por el reactor 2 y 1 cuyos porcentajes de remoción de DQO fueron 86 y $80 \%$ respectivamente. Conclusiones: El uso de microalga Chlorella sp. puede utilizarse en la eliminación de materia orgánica expresada en DQO de los efluentes de remojo de curtiduría.

Palabras clave: microalga Chlorella sp., materia orgánica, efluente, curtiembre, DQO.

\section{ABSTRACT}

Objective: Evaluate on scale laboratory the removal of the DQO using microalga Chlorella saccharophila live suspended, for the treatment of tannery wastewater from soaking stage. Material and Methods: Three bioreactors were used, which allowed the entry of air and sunlight, in which different volume relationships were established between the microalgae solution and the soaking water equivalent to $3: 1,1: 1$ and 1:3, taking samples every 2 days for its subsequent analysis of DQO in the Water Research Laboratory of the National University of Trujillo. Results: The results show that the 1:3 volume ratio of the reactor is the one that managed to remove the highest percentage of COD reaching $90 \%$ on day 14 of treatment, followed by reactor 2 and 1 , whose percentage removal of COD were 86 and $80 \%$ respectively. Conclusions: The use of microalga chlorella sp. can be used in the removal of organic matter expressed in COD of soaking effluent of a tannery.

Key word: microalga chlorella sp., organic matter, effluent, tannery.

\footnotetext{
${ }^{1}$ Universidad Nacional de Trujillo. Trujillo, Perú.

${ }^{2}$ Universidad Nacional José Faustino Sánchez Carrión. Huacho, Perú.
} 


\section{INTRODUCCIÓN}

El curtido de pieles es el proceso químico mediante el cual se convierten las pieles crudas de ovino o vacuno en cuero terminado.

Siendo considerada como una industria altamente contaminante debido que sus actividades consumen elevadas cantidades de agua en sus procesos; generando aguas residuales con elevadas cantidades de carga orgánica (demanda química y biológica de oxígeno), materia inorgánica, sulfuro, cromo, sólidos disueltos, sólidos en suspensión, nitrógeno orgánico entre otros, originando una creciente preocupación medioambiental en cuanto a su liberación (Esparza y Gamboa, 2001 y Dixit, Yadav, Dwivedi y Das, 2015). El tratamiento de aguas residuales con cultivos de microalgas es una tecnología eco-amigable, ya que reciclan eficientemente los residuos contaminantes de las aguas residuales.

Las microalgas son ampliamente utilizadas en el tratamiento de efluentes ya sean domésticos como industriales ya que estos desechos son un medio adecuado que favorece la proliferación rápida, gracias a su tolerancia a elevadas concentraciones de nutrientes $(N$ y $P$ ) y materia orgánica (Pérez y Labbé, 2014 y Vacca-Jimeno, AnguloMercado, Puentes-Ballesteros, y Plaza-Vega, 2017).

En la presente investigación se evaluó la capacidad de la microalga Chlorella sp., en la remoción de materia orgánica como demanda química de oxígeno de los efluentes de remojo de la industria de curtiembre; estos efluentes de remojo son generados en la etapa de ribera, las cuales contienen elevadas cargas orgánicas como DBO y DQO libre de cromo debido a que es una etapa anterior al curtido al cromo.

\section{MATERIALY MÉTODOS}

La solución de microalgal que se utilizó en el estudio consistió en un cultivo de microalgas del tipo Chlorella saccharophila. Inicialmente cultivadas en condiciones de luz natural y temperatura ambiente.
Los efluentes de remojo proceden de la curtiembre Ecológica del Norte, ubicada en el Parque Industrial del distrito de la Esperanza en la ciudad de Trujillo. Las muestras fueron trasladadas al Laboratorio en recipientes oscuros y cerrados.

Se diseñaron 4 biorreactores plásticos tipo BATCH de 3 litros de capacidad operados en condiciones de aireación permanente y luz solar natural.

Los reactores tuvieron diferentes relaciones de volúmenes, equivalentes a 3:1,1:1 y 1:3, con respecto a la solución de microalga y agua de remojo.

Se tomaron muestras de cada biorreactor a una hora definida a intervalos de 2 días, hasta los 14 días de tratamiento, para su posterior análisis de DQO; según la metodología descrita en Métodos Normalizados para el análisis de Aguas potables y Residuales (APHA, AWWA y WPCF, 1998).

\section{RESULTADOS}

El efluente de remojo presentó valores iniciales de pH y de DQO de 9,6 y 14083 ppm respectivamente.

En la Tabla 1 y Figura 1 se muestran los valores de DQO obtenidos en los diferentes tratamientos, donde se observa una disminución en la DQO muy pronunciada hasta el día 6 en todos los tratamientos, para luego ser muy lenta la remoción de materia orgánica hasta llegar a estabilizarse en el día 11 ; el nitrógeno y el fósforo son los principales nutrientes necesarios para el crecimiento de las microalgas (Usha, Sarat Chandra, Sarada y Chauhan, 2016).

La Figura 2 muestra los porcentajes de remoción de DQO de los diferentes tratamientos comparando la variación en el tiempo, hasta los 14 días de tratamiento. 
Tabla 1. Valores de $D Q O$ de los diferentes tratamientos obtenidos cada 2 días

\begin{tabular}{cccccccccccc}
\hline \multirow{2}{*}{ REACTOR } & \multicolumn{2}{c}{ Relación } & \multicolumn{10}{c}{ Días / DQO (mg/L) } \\
\cline { 2 - 12 } & Microalga & $\begin{array}{c}\text { Agua de } \\
\text { Remojo }\end{array}$ & 0 & 2 & 4 & 6 & 8 & 10 & 12 & 14 \\
\hline 1 & 3 & 1 & 4425 & 4047 & 2340 & 1016 & 1091 & 1048 & 1045 & 1021 \\
2 & 1 & 1 & 7950 & 6187 & 3788 & 2076 & 1370 & 1169 & 1109 & 1093 \\
3 & 1 & 3 & 11475 & 10718 & 7485 & 3230 & 2282 & 1206 & 1181 & 1138 \\
\hline
\end{tabular}

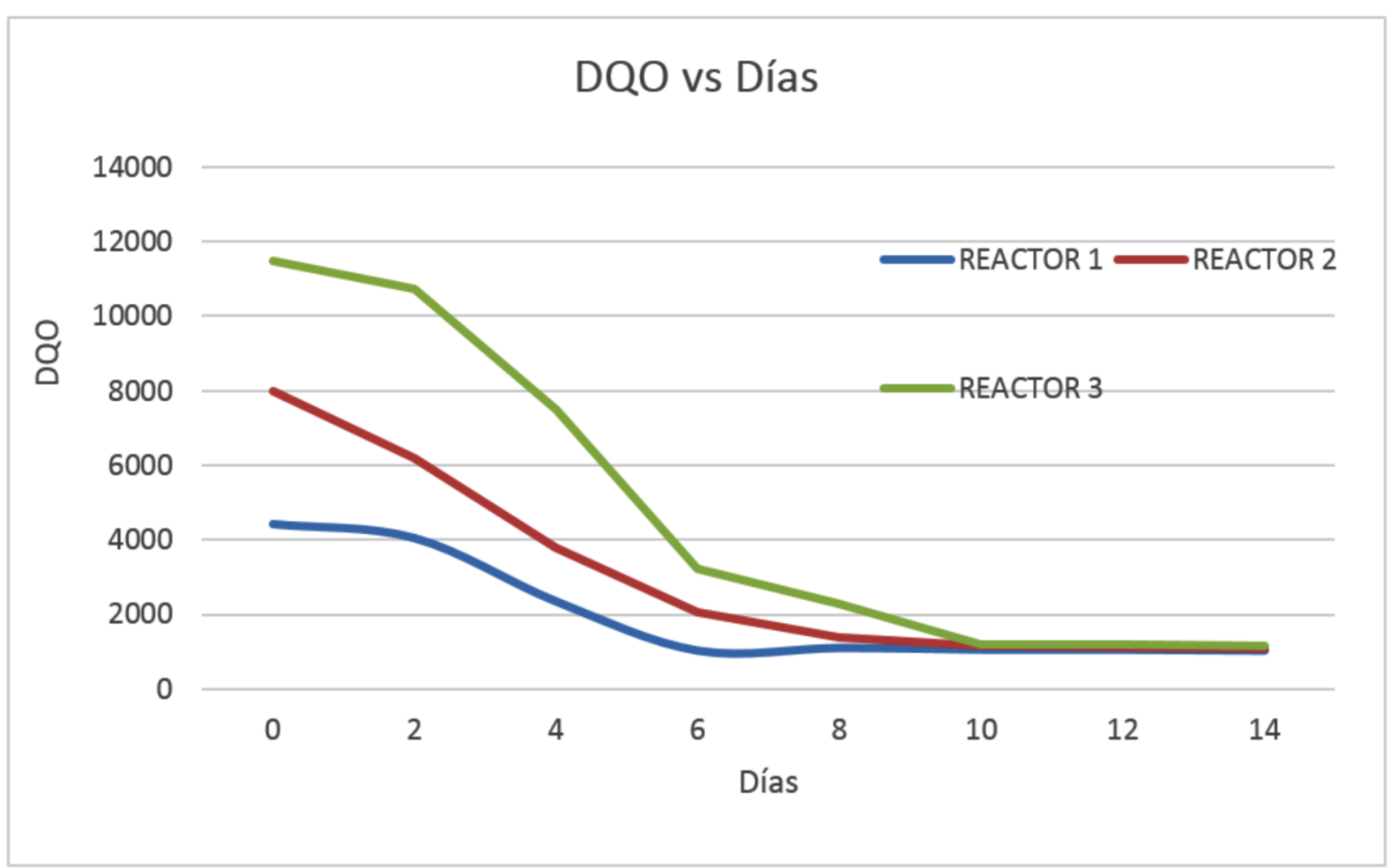

Figura 1. Variación de la $\mathrm{DQO}$ de los diferentes tratamientos evaluados cada 2 días

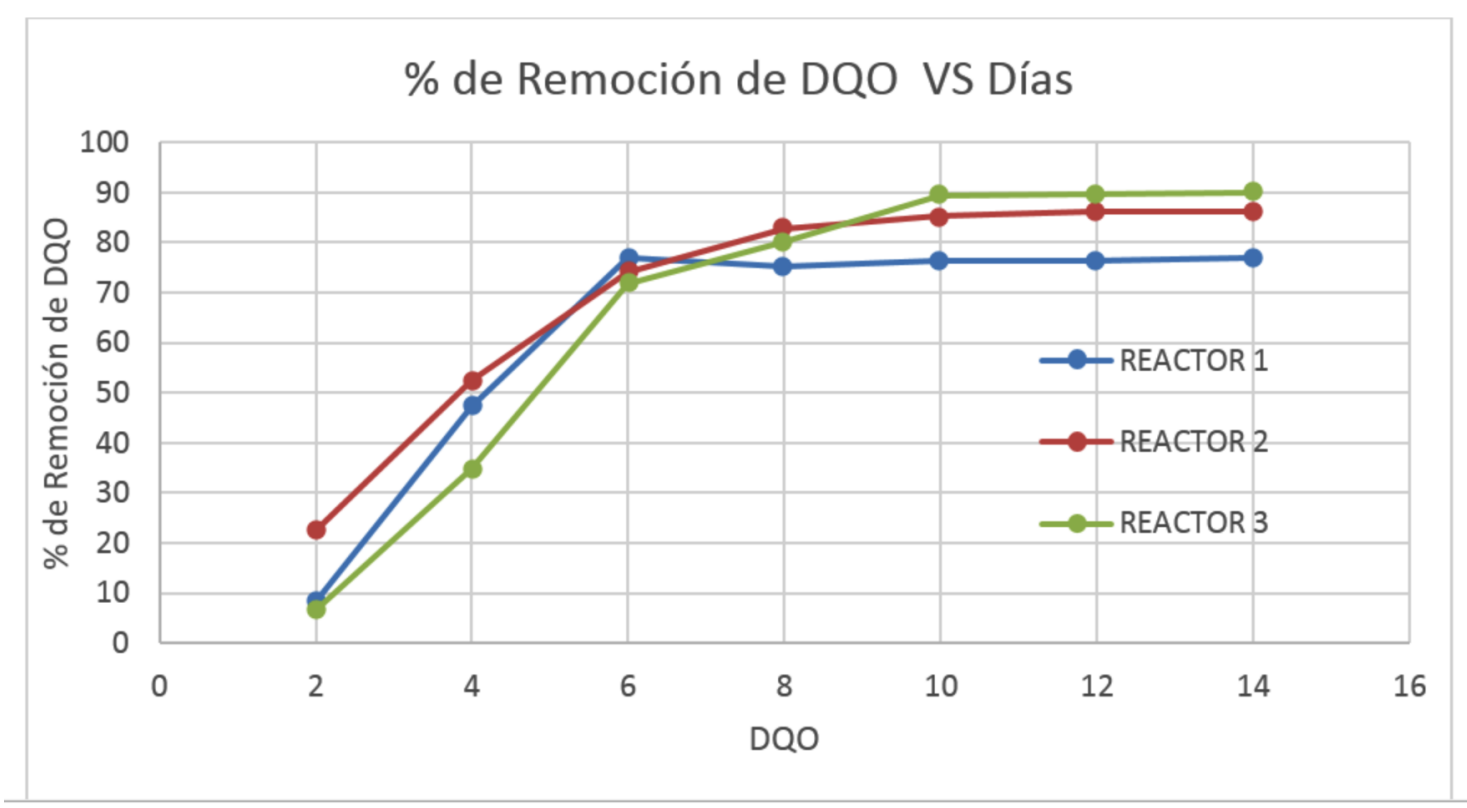

Figura 2. Variación del \% de remoción de DQO en el tiempo, evaluadas cada 2 días 


\section{DISCUSIÓN}

El valor de DQO inicial es muy elevado en comparación con lo exigido en la Normativa, Valor Máximo Admisible (PRODUCE, 2002) para descargas no domésticos de 1000 ppm de DQO. Existiendo la necesidad de realizar un tratamiento previo de adecuación de los efluentes de curtiembre antes de ser eliminados en el sistema de alcantarillado.

De la Figura 2 se observa que en la fase exponencial de crecimiento de las microalgas (días 2 al 6) se remueven la mayor parte de la DQO, tal es así que en el día 6 la remoción de DQO supera el $70 \%$ para los 3 tratamientos. Llegando a remover el $77 \%$ de la DQO en el reactor 1 que contenía 3 volúmenes de solución microalgal y 1 volumen de agua de Remojo; disminuyendo el valor de DQO desde 4425 hasta un valor de 1016 ppm. La misma tendencia se muestra en el reactor 3 el cual contenía 3 volúmenes de agua de remojo y un volumen de solución microalgal, llegando a remover el $72 \%$ de la DQO presente.

A partir del día 10 el aumento del porcentaje de remoción de DQO no es significativo para ningún tratamiento, siendo las modificaciones en el valor DQO mínimas tal como se muestra en la Tabla 1 y Figuras 1 y 2 , alcanzando valores finales de DQO en el día 14 de 890; 1 093 y 1138 ppm para los reactores 1, 2 y 3 respectivamente.

El reactor 3 que contiene relación de solución microalgal y agua de remojo de 1:3 respectivamente es el que logró remover el mayor porcentaje de DQO llegando a $90 \%$ en el día 14 de tratamiento, seguido por el reactor 2 y 1 cuyos porcentajes de remoción de DQO fueron 86 y 80 respectivamente al día 14 de tratamiento.

Las microalgas tienen un alto potencial para eliminar nutrientes de las aguas residuales y el cultivo de microalgas en las aguas residuales es un enfoque rentable para la remediación de la carga orgánica y los nutrientes. La eficacia del tratamiento de efluentes por microalgas está relacionada con su capacidad para reducir los niveles de DQO, DBO, nitrógeno y fósforo en el efluente (Usha et al., 2016).
El estudio permitió evaluar la utilización de microalga Chlorella sp. en la eliminación de la carga orgánica del efluente de remojo de curtiduría. Logrando eliminar más del $80 \%$ de la DQO al final de los 14 días de tratamiento.

El reactor 3 que contiene una relación de solución microalgal y agua de remojo de 1:3 respectivamente es el que logró remover el mayor porcentaje de DQO llegando a $90 \%$ en el día 14 de tratamiento, seguido por el reactor 2 y 1 cuyos porcentajes de remoción de DQO fueron 86 y $80 \%$ respectivamente al día 14 de tratamiento.

Por lo tanto, el presente estudio demostró que las microalgas Chlorella sp. se pueden utilizar eficazmente en la eliminación de materia orgánica expresada en DQO de los efluentes de remojo de curtiduría.

\section{REFERENCIAS BIBLIOGRÁFICAS}

APHA, AWWA \& WPCF. (1998). Standard Methods for the Examination of Water and Wastewater. 20th Edition. American Public Health Association 1015 Fifteenth Street, N.W. Washington, D.C.USA. 981 pp.

Dixit, S., Yadav, A., Dwivedi, P., \& Das, M. (2015). Toxic hazards of leather industry and technologies to combat threat: a review. Journal of Cleaner Production, 87, 39-49.

Esparza, E. \& Gamboa, N. (2001). Contaminación debida a la Industria de Curtiembre. Revista de Química Vol. $X V . N^{\circ}$ I. Pontificia Universidad Católica del Perú.

Pérez, Alexis \& Labbé, J. I. (2014). Microalgae, culture and benefits. Revista de Biología Marina y Oceanografía. Vol. 49,157-173.

Vacca-Jimeno, Víctor A., Angulo-Mercado, Edgardo R., Puentes-Ballesteros, Diana M., Torres-Yépez, José G., \& Plaza-Vega, Martin E. (2017). Uso de la microalga Chlorella sp. viva en suspensión en la decoloración del 
agua residual de una empresa textil. Prospectiva, 15 (1), 93-99. https://dx.doi.org/10.15665/rp.v15i1.8 29.

PRODUCE (2002, octubre, 04). D.S. NN 0032002-PRODUCE. Aprueban Límites Máximos Permisibles y Valores Referenciales para las actividades industriales de cemento, cerveza y papel. Diario Oficial El Peruano, 230921 - 230925.
Usha, M.T., Sarat Chandra, T., Sarada, R. \& Chauhan, V.S. (2016). Removal of nutrients and organic pollution load from pulp and paper mill effluent by microalgae in outdoor open pond, Bioresource Technology. doi: http://dx.doi.org/10.1016/j.biortech.20 16.04.060.

Correo electrónico: jlmb76@hotmail.com

Revisión de pares:

Recibido: 11-09-2018

Aprobado: 13-12-2018 